\title{
The Combinatorics of Conflicts between Clauses
}

\author{
Oliver Kullmann \\ Computer Science Department \\ University of Wales Swansea \\ Swansea, SA2 8PP, UK \\ 0. Kullmann@Swansea.ac.uk \\ http://cs-svr1.swan.ac.uk/ csoliver/
}

\begin{abstract}
We study the symmetric conflict matrix of a multi-clause-set, where the entry at position $(i, j)$ is the number of clashes between clauses $i$ and $j$. The conflict matrix of a multi-clause-set, either interpreted as a multi-graph or a distance matrix, yields a new link between investigations on the satisfiability problem (in a wider sense) and investigations on the biclique partition number and on the addressing problem (introduced by Graham and Pollak) in graph theory and combinatorics. An application is given by the well-known class of what are called 1-uniform hitting clause-sets in this article, where each pair of (different) clauses clash in exactly one literal. Endre Boros conjectured at the SAT'98 workshop that each 1-uniform hitting clause-set of deficiency 1 contains a literal occurring only once. Kleine Büning and Zhao showed, that under this assumption every 1-uniform hitting clause-set must have deficiency at most 1 . We show that the conjecture is false (there are known starfree biclique decompositions of every complete graph with at least nine vertices), but the conclusion is right (a special case of the Graham-Pollak theorem, attributed to Witsenhausen). A basic notion for investigations on the combinatorics of clause-sets is the deficiency of multi-clause-sets (the difference of the number of clauses and the number of variables). We introduce the related notion of hermitian defect, based on the notion of the hermitian rank of a hermitian matrix introduced by Gregory, Watts and Shader. The notion of hermitian defect makes it possible to combine eigenvalue techniques (especially Cauchy's interlacing inequalities) with matching techniques, and can be seen as the underlying subject of our introduction into the subject.
\end{abstract}

\section{Introduction}

Consider a multi-clause-set $F$ and its clause-variable matrix $M(F)$ (a matrix over $\{-1,0,+1\}$, where each row represents a clause). Using $|M(F)|$ for the matrix obtained from $M(F)$ by taking absolute values for each entry, we see

$$
|M(F)| \cdot|M(F)|^{t}-M(F) \cdot M(F)^{t}=2 \cdot \mathrm{C}_{\mathrm{s}}(F),
$$

where $A^{t}$ is the transposition of matrix $A$, and $\mathrm{C}_{\mathrm{s}}(F)$ is the symmetric conflict matrix of $F$, where the entry at position $(i, j)$ is the number of clashing literals 
between clauses $i$ and $j$ of $F$. It follows, that the number of variables in $F$ must be at least the maximum of the number of positive and the number of negative eigenvalues of $\mathrm{C}_{\mathrm{s}}(F)$ - the number of variables, which is the number of columns of $M(F)$, is a kind of a bottleneck in the above matrix multiplication; see Lemma 4 together with Lemma 5. For uniform hitting clause-sets with $m$ clauses, defined as (multi-)clause-sets $F$ where the entries of $\mathrm{C}_{\mathrm{s}}(F)$ are constant except of the diagonal (which is always zero), the symmetric conflict matrix has $m-1$ negative eigenvalues and one positive eigenvalue, and we conclude that every uniform hitting clause-set must have at most one clause more than it has variables (i.e., its deficiency is at most one). This observation is attributed to Witsenhausen by Graham and Pollak (9]), and has been the starting point for many investigations in graph theory and combinatorics. We review the basic notions and techniques, but formulated in the language of (multi-)clause-sets, which turns out to be a natural language for this kind of problems, providing a unifying point of view.

The addressing problem of Graham and Pollak (see Chapter 9 in [23]) considers a graph $G$ and its distance matrix $A$, which is a square matrix of order $|V(G)|$ with the distance in $G$ between node $i$ and node $j$ at position $(i, j)$. The problem is to attach "addresses" to the nodes of $G$, which are tuples from $\{0,1, *\}^{n}$ for some $n$ to be minimised, such that the distance between nodes $i$ and $j$ equals the "distance" between their addresses, where the distance of two addresses is computed by first removing all positions where at least one of the two addresses has $\mathrm{a} *$, and then taking the Hamming distance of the remaining $\{0,1\}$-vectors. Identifying the positions in the address vectors with variables, and interpreting 0 as "positive", 1 as "negative" and * as "not there" we see, that an addressing of $G$ corresponds to a multi-clause-set $F \in \mathcal{M C} \mathcal{L} \mathcal{S}$ with $\mathrm{C}_{\mathrm{s}}(F)=A$. For example, 1-uniform hitting clause-sets with $m$ clauses correspond to addressings of the complete graph $K_{m}$. Interpreting addresses (in our terminology, clauses) as code words, the address problem has been introduced into coding theory in [22].

Given a multigraph $G$, a biclique partition is a list of edge-disjoint sub-graphs of $G$ (not necessarily induced) covering together all edges of $G$, such that each of these sub-graphs is a complete bipartite graph. Using the adjacency matrix $A$ of $G$ each biclique decomposition corresponds to a multi-clause-set with symmetric conflict matrix $A$ - each node is a clause, each biclique represents a variable, where the "left side" stands for the positive occurrences, while the "right side" stands for the negative occurrences, and each edge represents a conflict. 1-uniform hitting clause-sets correspond to biclique partitions of the complete graph (again). For literature see [15].

This article seeks to present an introduction into a new area of research, and nearly no proofs are given, but all proofs can be found in the underlying report [15. The organisation is as follows: After presenting some notations, in Section 3 we lay the foundations by investigating conflict matrices and their properties, most notable the hermitian rank and the classes of conflict matrices with bounded hermitian defect, where the hermitian defect is an upper bound for the maximal deficiency. Then in Section 4 we collect observations and results 
on hitting clause-sets, and apply our general results derived from the study of conflict matrices. Finally in Section 5 a counterexample to the conjecture of Endre Boros is discussed. Some main results are as follows:

1. In Subsection 3.3 the central (and novel) notion of hermitian defect is introduced, the difference of the order of a symmetric matrix and its hermitian rank. Strengthening the Graham-Pollak theorem by using interlacing inequalities, in Theorem 11 we obtain the central result of this section, that for every multi-clause-set $F$ and every strict sub-multi-clause-set $F^{\prime}$ the deficiency of $F^{\prime}$ is strictly less than the hermitian defect of $F$ (while the deficiency of $F$ itself is less or equal to the hermitian defect).

2. In Subsection 3.4 exact multi-clause-sets are introduced, which realise the symmetric conflict number of its symmetric conflict matrix. While deciding exactness of multi-clause-sets is co-NP complete, the strengthening of this notion by the notion of eigensharp multi-clause-sets is decidable in polynomial time, where a clause-set is eigensharp if its hermitian defect coincides with its deficiency. From our central result on the hermitian defect we obtain immediately, that every eigensharp multi-clause-set is matching lean, i.e., has no non-trivial matching autarky (Theorem 14).

3. The class $\mathcal{M C} \mathcal{L S}_{\delta_{\mathrm{h}}}(1)$ of multi-clause-sets with hermitian defect at most one is investigated in Subsection 3.5. Theorem 19 completely characterises eigensharp, matching lean and matching satisfiable multi-clause-sets $F \in$ $\mathcal{M C} \mathcal{L S}_{\delta_{\mathrm{h}}}(1)$, where it turns out, that the necessary condition $\delta(F) \geq 1$ (i.e., $\delta(F)=1)$ for the first two cases respectively $\delta(F) \leq 0$ for matching satisfiability are also sufficient. In Theorem 26 we show, that unsatisfiable multiclause-sets in $\mathcal{M C} \mathcal{L S}_{\delta_{\mathrm{h}}}(1)$ are exactly the saturated minimally unsatisfiable clause-sets of deficiency one.

4. In Subsection 3.6 the classes $\mathcal{M C L S}_{i_{+}}(k)$ and $\mathcal{M C} \mathcal{L S}_{i_{-}}(k)$ of multi-clausesets are introduced, where the symmetric conflict matrix has at most $k$ positive respectively negative eigenvalues. Again these classes are stable under formation of sub-multi-clause-sets and under application of partial assignments (Lemma 28). Theorem 29 applies the Perron-Frobenius theorem to show, that the largest eigenvalue of the symmetric conflict matrix of a multi-clause-set is also largest with respect to its absolute value. It follows $\mathcal{M C} \mathcal{L S}_{\delta_{\mathrm{h}}}(1) \subset \mathcal{M C} \mathcal{L} \mathcal{S}_{i_{+}}(1)$ (Theorem 30 ).

5. Hitting clause-sets are studied in Section 4 (every two clauses clash), and a fair amount of different characterisations and properties are given. Theorem 32 shows, that the class of unsatisfiable hitting clause-sets is exactly the class of all minimally unsatisfiable clause-sets which stay minimally unsatisfiable under applications of any partial assignments. For uniform hitting clausesets (every two clauses clash in the same number of literals) it is shown

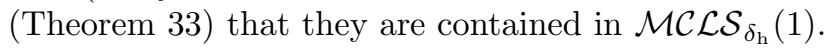

6. A counterexample to the conjecture of Endre Boros is given in Section 5

For all proofs see [15], where also a list of open problems is given (see [16] for the status of these (and other) open problems). 


\section{Some notations}

We use $\mathbb{N}=\{1,2, \ldots\}$ and $\mathbb{N}_{0}=\mathbb{N} \cup\{0\}$. The universe of variables is denoted by $\mathcal{V A}$. Clauses are finite and complement-free sets of literals, the set of all clauses is denoted by $\mathcal{C} \mathcal{L}$. The empty clause is denoted by $\perp:=\emptyset \in \mathcal{C} \mathcal{L S}$. A (finite) multiclause-set is a map $F: \mathcal{C} \mathcal{L} \rightarrow \mathbb{N}_{0}$ such that only for finitely many clauses $C$ we have $F(C) \neq 0$; the set of all multi-clause-sets is denoted by $\mathcal{M C} \mathcal{L} \mathcal{S}$. The empty multi-clause-set is denoted by $\top:=(0)_{C \in \mathcal{C} \mathcal{L}} \in \mathcal{M C} \mathcal{L} \mathcal{S}$. For $F, F^{\prime} \in \mathcal{M C} \mathcal{L} \mathcal{S}$ the relation $\boldsymbol{F} \leq \boldsymbol{F}^{\prime}$ holds iff for all $C \in \mathcal{C} \mathcal{L}$ we have $F(C) \leq F^{\prime}(C)$, while $\boldsymbol{F} \leq \boldsymbol{F}^{\prime}$ is equivalent to $F \leq F^{\prime}$ and $F \neq F^{\prime}$. The multi-clause-set $\boldsymbol{F}+\boldsymbol{F}^{\prime}$ is defined via $\left(F+F^{\prime}\right)(C):=F(C)+F^{\prime}(C)$. The number of clauses in $F$ is defined as $\boldsymbol{c}(\boldsymbol{F}):=\sum_{C \in \mathcal{C} \mathcal{L}} F(C)$, while the number of occurrences of a literal $x$ in $F$ is denoted by $\#_{x}(\boldsymbol{F}):=\sum_{C \in \mathcal{C} \mathcal{L}, x \in C} F(C)$. The set of variables occurring in $F$ is $\operatorname{var}(\boldsymbol{F}):=\bigcup_{C \in \mathcal{C}, F(C) \neq 0} \operatorname{var}(C)$, and the number of variables in $F$ is $\boldsymbol{n}(\boldsymbol{F}):=|\operatorname{var}(F)|$. For $F \in \mathcal{M C} \mathcal{L S}$ we have $n(F)=0$ iff for all clauses $C \in \mathcal{C} \mathcal{L} \backslash\{\perp\}$ we have $F(C)=0$. The deficiency of $F$ is $\boldsymbol{\delta}(\boldsymbol{F}):=c(F)-n(F)$, while the maximal deficiency is $\boldsymbol{\delta}^{*}(\boldsymbol{F}):=\max _{F^{\prime} \leq F} \delta\left(F^{\prime}\right)$. Due to $\delta(\top)=0$ we have $\delta^{*}(F) \geq 0$. $\mathcal{P A S S}$ denotes the set of all partial assignments, which are maps $\varphi: V \rightarrow\{0,1\}$ for finite set $V$ of variables. The domain of a partial assignment $\varphi$ is denoted by $\operatorname{var}(\varphi):=V$, while the size of the domain is denoted by $\boldsymbol{n}(\varphi):=|\operatorname{var}(\varphi)|$. The application of partial assignments $\varphi \in \mathcal{P A S S}$ to multiclause-sets $F \in \mathcal{M C L S}$ is denoted by $\varphi * \boldsymbol{F} \in \mathcal{M C L S}$, where for $C \in \mathcal{C} \mathcal{L}$ we have

$$
(\varphi * F)(C)=\sum_{\substack{C^{\prime} \in \mathcal{C} \mathcal{L} \\ \varphi *\left\{C^{\prime}\right\}=\{C\}}} F\left(C^{\prime}\right),
$$

where $\varphi *\left\{C^{\prime}\right\}$ is the empty set in case $\varphi$ satisfies $C$, while otherwise $\varphi *\left\{C^{\prime}\right\}=$ $\left\{C^{\prime \prime}\right\}$, where $C^{\prime \prime}$ is obtained from $C^{\prime}$ by removing all literals whose variables are in the domain of $\varphi$. Clause-sets, that is, finite sets of clauses, can be identified with multi-clause-sets $F: \mathcal{C} \mathcal{L} \rightarrow\{0,1\}$. The set of all clause-sets is denoted by $\mathcal{C} \mathcal{L} \mathcal{S}$, and the operation of $\mathcal{P} A \mathcal{S} \mathcal{S}$ on $\mathcal{C} \mathcal{L S}$ is defined as via $\varphi * F:=\hat{\mathrm{t}}(\varphi * F)$ for $F \in \mathcal{C} \mathcal{L S}$ and $\varphi \in \mathcal{P A S S}$, where $\hat{\mathrm{t}}: \mathcal{M C} \mathcal{L S} \rightarrow \mathcal{C} \mathcal{L S}$ is the canonical projection morphism, which contracts multiple clause occurrences. For $F \in \mathcal{M C L S}$ and a clause-set $F^{\prime} \in \mathcal{C} \mathcal{L S}$ we define $\boldsymbol{F} \backslash \boldsymbol{F}^{\prime} \in \mathcal{M C L S}$ by $\left(F \backslash F^{\prime}\right)(C):=F(C)$ for

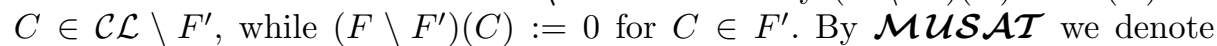
the class of minimally unsatisfiable clause-sets, while by $\mathcal{S} \boldsymbol{M U \mathcal { S A T }}$ we denote the class of saturated minimally unsatisfiable clause-set, which are minimally unsatisfiable clause-sets which become satisfiable when any literal is added to any clause (another notion used for example in [3] instead of "saturated" is "maximal"). For any class $\mathcal{C} \subseteq \mathcal{M C} \mathcal{L S}$ of multi-clause-sets and any measure $f: \mathcal{M C} \mathcal{L} \mathcal{S} \rightarrow \mathbb{N}_{0}$ we define for $k \in \mathbb{N}_{0}$

$$
\mathcal{C}_{f}(k):=\{F \in \mathcal{C}: f(F) \leq k\} .
$$

It follows that for example $\mathcal{M U S} \mathcal{S} \mathcal{T}_{\delta}(1)$ is the class of minimally unsatisfiable clause-sets of deficiency 1. 
The clause-variable matrix $\boldsymbol{M ( F )}$ for $F \in \mathcal{M C} \mathcal{L} \mathcal{S}$ is a $c(F) \times n(F)$-matrix over $\{-1,0,+1\}$. The order of rows and columns of $M(F)$ is arbitrary, but fixed in any given context. The matrix $\boldsymbol{E}_{\boldsymbol{m}, \boldsymbol{i}, \boldsymbol{j}}$ is the square matrix of order $m$ with entry 1 at position $(i, j)$ and 0 elsewhere. Furthermore we use $\boldsymbol{J}_{\boldsymbol{m}}:=$ $\sum_{i=1}^{m} \sum_{j=1}^{m} E_{m, i, j}$ for the all-one matrix, while the identity matrix is denoted by $\boldsymbol{I}_{\boldsymbol{m}}:=\sum_{i=1}^{m} E_{m, i, i}$. The transposition of a matrix $A$ is denoted by $\boldsymbol{A}^{\boldsymbol{t}}$. We consider $m \times n$-matrices for $m, n \in \mathbb{N}_{0}$; for example the $m \times 0$-matrix is the clausevariable matrix of the multi-clause-set $F$ given by $F(\perp)=m$ and $F(C)=0$ for $C \in \mathcal{C} \mathcal{L} \backslash\{\perp\}$. If we speak in this article of a graph respectively of a directed graph, we mean a simple undirected graph respectively a simple directed graph (without loops and without parallel edges), while a (directed) multigraph is still loopless, but may have parallel edges.

\section{Conflict matrices}

A conflict matrix is a square matrix of order $m \in \mathbb{N}_{0}$ with entries in $\mathbb{N}_{0}$ and with zeros on the main diagonal (in other words, a conflict matrix is the adjacency matrix of some directed multigraph). A symmetric conflict matrix is a conflict matrix which is symmetric (in other words, symmetric conflict matrices are the adjacency matrices of multigraphs). Addition of (symmetric) conflict matrices yields again a (symmetric) conflict matrix. For $F \in \mathcal{M C L S}$ let $\mathbf{C}_{\mathbf{s}}(\boldsymbol{F})$ be the symmetric conflict matrix of $F$, which is a square matrix of order $c(F)$ with entries $\mathrm{C}_{\mathrm{s}}(F)_{i, j}:=\left|C_{i} \cap \overline{C_{j}}\right|$ (the number of literals $x \in C_{i}$ with $\bar{x} \in C_{j}$ ), where the $C_{i}$ are the clauses of $F$ (in some order; with multiplicities). And let $\mathbf{C}_{\mathbf{a}}(\boldsymbol{F})$ be the asymmetric conflict matrix of $F$, a square matrix of order $c(F)$ with entries $\mathrm{C}_{\mathrm{a}}(F)_{i, j}:=\left|\left(C_{i} \cap \mathcal{V} \mathcal{A}\right) \cap \overline{C_{j}}\right|$ (the number of positive literals $x \in C_{i}$ with $\left.\bar{x} \in C_{j}\right)$. Obviously $\mathrm{C}_{\mathrm{s}}(F)$ is a symmetric conflict matrix, while $\mathrm{C}_{\mathrm{a}}(F)$ is a conflict matrix, and we have

$$
\mathrm{C}_{\mathrm{s}}(F)=\mathrm{C}_{\mathrm{a}}(F)+\mathrm{C}_{\mathrm{a}}(F)^{t} .
$$

Lemma 1. For every conflict matrix $A$ there is $F \in \mathcal{M C L S}$ with $\mathrm{C}_{\mathrm{a}}(F)=A$, and for every symmetric conflict matrix $A$ there is $F \in \mathcal{M C L S}$ with $\mathrm{C}_{\mathrm{s}}(F)=A$.

The underlying (trivial) construction is very wasteful with variables, and it is natural to seek to minimise the number of variables used in $F$, or, in other words, to maximise the deficiency of $F$. This problem is the underlying theme of investigations in this context, and will be studied from Subsection 3.1 on. Consider $F, F^{\prime} \in \mathcal{M C L S}$ with $F^{\prime} \leq F$. The symmetric conflict matrix $\mathrm{C}_{\mathrm{s}}\left(F^{\prime}\right)$ is a principal submatrix of $\mathrm{C}_{\mathrm{s}}(F)$, and the asymmetric conflict matrix $\mathrm{C}_{\mathrm{a}}\left(F^{\prime}\right)$ is a principal submatrix of $\mathrm{C}_{\mathrm{a}}(F)$ ("principal submatrices" are obtained by deletion of rows and columns with the same indices). Since application of partial assignments can be realised by first removing all clauses satisfied by the assignment, and then eliminating all remaining literal occurrences falsified by the assignment, where these occurrences have become pure, for any partial assignment $\varphi \in \mathcal{P} \mathcal{A S S}$ also $\mathrm{C}_{\mathrm{s}}(\varphi * F)$ resp. $\mathrm{C}_{\mathrm{a}}(\varphi * F)$ is a principal submatrix of $\mathrm{C}_{\mathrm{s}}(F)$ resp. $\mathrm{C}_{\mathrm{a}}(F)$. 
For a class $\mathcal{C}$ of symmetric conflict matrices stable under formation of principal submatrices let

$$
\mathcal{M C L S}(\mathcal{C}):=\left\{F \in \mathcal{M C L S}: \mathrm{C}_{\mathrm{s}}(F) \in \mathcal{C}\right\}
$$

be the set of multi-clause-sets with symmetric conflict matrix in $\mathcal{C}$. It follows that $\mathcal{M C} \mathcal{L} \mathcal{S}(\mathcal{C})$ is stable under sub-multi-clause-set formation and application of partial assignments. Examples for such classes $\mathcal{C}$ are given as the classes of all symmetric conflict matrices $A$ defined by one of the following conditions:

1. $A \geq J_{m}-I_{m}$ for arbitrary $m \in \mathbb{N}_{0}$; the corresponding multi-clause-sets are the hitting clause-sets considered in Section 4 ,

2. $A=k \cdot\left(J_{m}-I_{m}\right)$ for some fixed $k \in \mathbb{N}$ and arbitrary $m \in \mathbb{N}_{0}$; the corresponding $k$-uniform hitting clause-sets are considered in Section 4

3. A has no entry 1; the class of corresponding "resolution-free" clause-sets has been considered in [17;

4. $\delta_{\mathrm{h}}(A) \leq k$ for some fixed $k \in \mathbb{N}_{0}$ (see Subsection 3.5); the "hermitian defect" $\delta_{\mathrm{h}}(A)$ is introduced in Subsection 3.2, and gives an upper bound on the deficiency of multi-clause-sets $F$ with $\mathrm{C}_{\mathrm{s}}(F)=A$; we will see that $\delta_{\mathrm{h}}(k$. $\left.\left(J_{m}-I_{m}\right)\right)=1$ for $k, m \neq 0$ (cf. example 2 );

5. $i_{+}(A) \leq k$ resp. $i_{-}(A) \leq k$ for some $k \in \mathbb{N}_{0}$, where $i_{ \pm}(A)$ is the number of positive resp. negative eigenvalues of $A$ (see Subsection 3.6); we will see that $\delta_{\mathrm{h}}(A) \leq 1$ implies $i_{+}(A) \leq 1$.

\subsection{Symmetric and asymmetric conflict number}

For a symmetric conflict matrix $A$ let $\mathbf{n}_{\mathbf{s}}(\boldsymbol{A})$, the symmetric conflict number of $A$, be the minimal $n(F)$ for $F \in \mathcal{M C L \mathcal { S }}$ with $\mathrm{C}_{\mathrm{s}}(F)=A$. And for a conflict matrix $A$ let $\mathbf{n}_{\mathbf{a}}(\boldsymbol{A})$, the asymmetric conflict number of $A$, be the minimal $n(F)$ for $F \in \mathcal{M C L \mathcal { S }}$ with $\mathrm{C}_{\mathrm{a}}(F)=A$. Obviously we have $n(F) \geq \mathrm{n}_{\mathrm{a}}\left(\mathrm{C}_{\mathrm{a}}(F)\right) \geq$ $\mathrm{n}_{\mathrm{s}}\left(\mathrm{C}_{\mathrm{s}}(F)\right)$. For conflict matrices $A$ the asymmetric conflict number $\mathrm{n}_{\mathrm{a}}(A)$ is the biclique partition number of a directed multigraph with adjacency matrix $A$, while for symmetric conflict matrices $A$ the symmetric conflict number $\mathrm{n}_{\mathrm{s}}(A)$ is the biclique partition number of an undirected multigraph with adjacency matrix $A$. Thus we can apply [12] and conclude, that the decision problem $\mathrm{n}_{\mathrm{s}}(A) \leq k$, where the input size is $k$ plus the size of the graph with adjacency matrix $A$, is NP-complete.

Lemma 2. Consider a conflict matrix A.

1. The asymmetric conflict number $\mathrm{n}_{\mathrm{a}}(A)$ is the minimal $q \in \mathbb{N}_{0}$, such that there are $\{0,1\}$-matrices $B_{1}, \ldots, B_{q}$ of rank 1 with $A=B_{1}+\cdots+B_{q}$.

2. If $A$ is symmetric, then $\mathrm{n}_{\mathrm{s}}(A)$ is the minimal $q \in \mathbb{N}_{0}$, such that there are symmetric $\{0,1\}$-matrices $B_{1}, \ldots, B_{q}$ of rank 2 with $A=B_{1}+\cdots+B_{q}$.

Lemma 3. For every conflict matrix $A$ of order $m \in \mathbb{N}_{0}$ the asymmetric conflict number $\mathrm{n}_{\mathrm{a}}(A)$ is the minimal $q \in \mathbb{N}_{0}$ such that there is an $m \times q$-matrix $X$ and a $q \times$ m-matrix $Y$ over $\{0,1\}$ with $A=X \cdot Y$. 


\subsection{The hermitian rank}

Since every presentation $A=B+B^{t}$ for a symmetric conflict matrix $A$ and a conflict matrix $B$ corresponds to one possible choice for the polarities of conflicting (clashing) literals, the following lemma holds.

Lemma 4. For every symmetric conflict matrix $A$ the symmetric conflict number $\mathrm{n}_{\mathrm{s}}(A)$ equals the minimal asymmetric conflict number $\mathrm{n}_{\mathrm{a}}(B)$ for conflict matrices $B$ with $B+B^{t}=A$. Thus by Lemma 3 if follows that $\mathrm{n}_{\mathrm{s}}(A)$ is the minimal $q$ such that there are $\{0,1\}$-matrices $X, Y$ with $A=X Y+Y^{t} X^{t}$, where $X$ has $q$ columns and $Y$ has $q$ rows.

For any symmetric real matrix $A$ let $\boldsymbol{i}_{+}(\boldsymbol{A})$ be the number of positive eigenvalues of $A$, and let $\boldsymbol{i}_{-}(\boldsymbol{A})$ be the number of negative eigenvalues of $A$. Following [10], the hermitian rank $\boldsymbol{h}(\boldsymbol{A})$ of $A$ now is defined as

$$
h(A):=\max \left(i_{+}(A), i_{-}(A)\right) .
$$

See for example [18, Chapters 10 and 11 for background information on orthogonal geometries. The following characterisations of the hermitian rank are direct consequences of the results from [10].

Lemma 5. Consider a symmetric real matrix A. Now the following three quantities are equal to the hermitian rank $h(A)$ :

1. the minimal number $k \in \mathbb{N}_{0}$ such that there are real matrices $X, Y$ with $A=X^{t} X-Y^{t} Y$, where $X, Y$ both have $k$ rows;

2. the minimal number $k \in \mathbb{N}_{0}$ such that there are real matrices $X, Y$ with $A=Y^{t} X+X^{t} Y$, where $X, Y$ both have $k$ rows;

3. the minimal $\operatorname{rank}(B)$ for real matrices $B$ with $A=B+B^{t}$.

Corollary 6. For all symmetric real matrices $A, B$ we have $h(A+B) \leq h(A)+$ $h(B)$. It follows, that for $X=A+B$ we have $h(X) \geq h(A)-h(B)$.

\subsection{The hermitian defect}

Let $\boldsymbol{\delta}_{\mathrm{h}}(\boldsymbol{A}):=m-h(A)$ be the hermitian defect of a symmetric real matrix $A$ of order $m$, and for $F \in \mathcal{M C L S}$ let $\boldsymbol{\delta}_{\mathbf{h}}(\boldsymbol{F}):=\delta_{\mathrm{h}}\left(\mathrm{C}_{\mathrm{s}}(F)\right)$. For rational matrices $A$ the hermitian defect can be computed in polynomial time. Since a conflict matrix has trace zero, and the trace of a symmetric real matrix equals the sum of its eigenvalues (with multiplicities), for every non-empty conflict matrix $A$ we have $\delta_{\mathrm{h}}(A) \geq 1$. By Lemma 4 together with Lemma 5 the following reformulation of the Graham-Pollak Theorem is a direct consequence.

Corollary 7. For every conflict matrix $A$ we have $\mathrm{n}_{\mathrm{s}}(A) \geq h(A)$. It follows that for every multi-clause-set $F \in \mathcal{M C} \mathcal{L S}$ the deficiency of $F$ is at most the hermitian defect of $F$, i.e., $\delta(F) \leq \delta_{\mathrm{h}}(F)$. 
For a real symmetric matrix $A$ of order $m$ let $\theta_{1}(A) \geq \cdots \geq \theta_{m}(A)$ denote the eigenvalues of $A$ (in descending order). If $B$ is a real symmetric matrix of order $m^{\prime} \leq m$, then it is said that the eigenvalues of $B$ interlace the eigenvalues of $A$ iff we have (see Chapter 9 in [8])

$$
\begin{aligned}
& \theta_{1}(A) \geq \theta_{1}(B), \quad \theta_{2}(A) \geq \theta_{2}(B), \quad \ldots \quad \quad \theta_{m}(A) \geq \theta_{m}(B) \\
& \theta_{n}(A) \leq \theta_{m}(B), \quad \theta_{n-1}(A) \leq \theta_{m-1}(B), \quad \ldots \quad \theta_{n-m+1}(A) \leq \theta_{1}(B) .
\end{aligned}
$$

Lemma 8. Consider two real symmetric matrices $A, B$ such that the eigenvalues of $B$ interlace the eigenvalues of $A$. Then $\delta_{\mathrm{h}}(B) \leq \delta_{\mathrm{h}}(A)$ holds.

Since the eigenvalues of a principal submatrix of a symmetric real matrix $A$ interlace the eigenvalues of $A$ (see Theorem 9.1.1 in [8]), we obtain the following fundamental property of the hermitian rank.

Corollary 9. For every real symmetric matrix $A$ and every principal submatrix $A^{\prime}$ we have $\delta_{\mathrm{h}}\left(A^{\prime}\right) \leq \delta_{\mathrm{h}}(A)$. It follows that for every $F, F^{\prime} \in \mathcal{M C} \mathcal{L S}$ with $F^{\prime} \leq F$ and every partial assignment $\varphi$ we have $\delta_{\mathrm{h}}\left(F^{\prime}\right) \leq \delta_{\mathrm{h}}(F)$ and $\delta_{\mathrm{h}}(\varphi * F) \leq \delta_{\mathrm{h}}(F)$.

Corollary 10. $\forall F \in \mathcal{M C} \mathcal{L} S: \delta^{*}(F) \leq \delta_{\mathrm{h}}(F)$.

A further strengthening is possible (compare [1]).

Theorem 11. For all $F, F^{\prime} \in \mathcal{M C} \mathcal{L S}$ with $F^{\prime} \lesseqgtr F$ we have $\delta\left(F^{\prime}\right)<\delta_{\mathrm{h}}(F)$.

Corollary 12. For multi-clause-sets $F \in \mathcal{M C L S}$ with $\delta(F) \leq \delta_{\mathrm{h}}(F)-1$ we have $\delta^{*}(F) \leq \delta_{\mathrm{h}}(F)-1$.

\subsection{Exact and eigensharp multi-clause-sets}

A conflict matrix $A$ is called eigensharp if $\mathrm{n}_{\mathrm{s}}(A)=h(A)$ holds. Thus a conflict matrix $A$ is eigensharp iff there is $F \in \mathcal{M C L \mathcal { S }}$ with $\mathrm{C}_{\mathrm{s}}(F)=A$ and $\delta(F)=$ $\delta_{\mathrm{h}}(F)=\delta_{\mathrm{h}}(A)$. Every zero matrix $0 \cdot J_{m}$ is eigensharp. The notion "eigensharp" was introduced in [12] for biclique partitions of graphs; it seems more natural to put the matrix $A$ into the foreground, while the graph with adjacency matrix $A$ is only one possible representation of $A$ - another possible representation is to consider a graph such that its distance matrix is $A$. To decide whether $F \in$ $\mathcal{M C} \mathcal{L} \mathcal{S}$ or a symmetric conflict matrix is eigensharp can be done in polynomial time. Call $F \in \mathcal{M C} \mathcal{L S}$ exact if $n(F)=\mathrm{n}_{\mathrm{s}}\left(\mathrm{C}_{\mathrm{s}}(F)\right)$ holds, and call $F$ eigensharp if $\delta(F)=\delta_{\mathrm{h}}(F)$ holds. Thus $F$ is eigensharp iff $F$ is exact and $\mathrm{C}_{\mathrm{s}}(A)$ is eigensharp. Every $F \in \mathcal{M C L S}$ with $n(F)=0$ is eigensharp, and if $\mathrm{C}_{\mathrm{s}}(F)$ has at least one entry equal to $n(F)$ then $F$ must be exact. Eigensharp multi-clause-sets correspond to eigensharp addressings as introduced in [5], while exact multiclause-sets have been called optimal addressings in [5]; the term "exact" is taken from the literature on biclique decompositions. It seems worth to note, that in our context a certain shift of attention takes place: While in [5] an eigensharp addressing is attached to a graph (i.e., distance matrix), in our context the multiclause-set (addressing) is more dominant, and the distance matrix is derived from it. To decide whether $F \in \mathcal{M C} \mathcal{L} \mathcal{S}$ is exact is co-NP complete. 
Lemma 13. If a multi-clause-set $F \in \mathcal{M C L S}$ is exact, then $F$ does not contain pure literals, and if $F$ is eigensharp and $F \neq \top$, then we have $\delta(F) \geq 1$.

For exact multi-clause-sets $F$ in general $\delta(F) \geq 1$ need not hold; e.g. $F=$ $\left\{\left\{v_{1}, \ldots, v_{k}\right\},\left\{\overline{v_{1}}, \ldots, \bar{v}_{k}\right\}\right\}$ is exact with $\delta(F)=2-k$. However, if $F$ is exact and $\mathrm{C}_{\mathrm{s}}(F)$ is the distance matrix of a non-empty connected graph, then Winkler proved $\delta(F) \geq 1$ (Theorem 15). We seek to generalise this result. In 14 the notion of matching lean clause-sets has been introduced, and the equivalence of the following conditions for $F \in \mathcal{M C L S}$ have been proven (for us Criterion 3 can be taken as a definition of matching leanness):

1. $F$ is matching lean.

2. $F$ has no non-trivial matching autarky.

3. For all $F^{\prime} \in \mathcal{M C} \mathcal{L S}$ with $F^{\prime} \lesseqgtr F$ we have $\delta\left(F^{\prime}\right)<\delta(F)$.

Theorem 11 together with Criterion 3 immediately yields

Theorem 14. Every eigensharp multi-clause-set is matching lean.

A matrix is called a distance matrix if it is the distance matrix of some finite metric space. The following result of P. Winkler (see Chapter 9 in [23]) considers special distance matrices, where the underlying metric space is given by the distances of nodes in some (undirected) graph.

Theorem 15. For every exact multi-clause-set $F$, where $\mathrm{C}_{\mathrm{s}}(F)$ is the distance matrix of some non-empty connected graph, we have $\delta(F) \geq 1$.

Conjecture 16. Every exact multi-clause-set $F \in \mathcal{M C} \mathcal{L S}$, where the symmetric conflict matrix $\mathrm{C}_{\mathrm{s}}(F)$ is the distance matrix of some connected graph, is matching lean.

\subsection{Multi-clause-sets with hermitian defect one}

For $k \in \mathbb{N}_{0}$ let $\mathcal{H} \mathcal{D}(\boldsymbol{k})$ be the set of all real symmetric $m \times m$-matrices $A$ for $m \in \mathbb{N}_{0}$ with zero diagonal and hermitian defect $\delta_{\mathrm{h}}(A) \leq k$. Thus we have $\mathcal{M C} \mathcal{L} \mathcal{S}_{\delta_{\mathrm{h}}}(k)=\mathcal{M C} \mathcal{L} \mathcal{S}(\mathcal{H} \mathcal{D}(k)) . \mathcal{M C} \mathcal{L} \mathcal{S}_{\delta_{\mathrm{h}}}(0)$ just contains the empty multiclause-set $T$. By Lemma 8 we get immediately the following closure properties.

Lemma 17. For all $k \in \mathbb{N}_{0}$ the set $\mathcal{H D}(k)$ is stable under formation of principal submatrices, and so is the subset of $\mathcal{H D}(k)$ consisting of all conflict matrices. Thus $\mathcal{M C L S}_{\delta_{\mathrm{h}}}(k)$ is stable under application of partial assignments and formation of sub-multi-clause-sets.

In 13 the classes $\mathcal{M C} \mathcal{L} \mathcal{S}_{\delta^{*}}(\boldsymbol{k})$ of multi-clause-sets with bounded maximal deficiency have been introduced, and it has been shown, that several hard problems like satisfiability decision or computation of the maximal autark subset are decidable for these classes in polynomial time; see 6] for an alternative proof of the main result, and see [21] for the fixed parameter tractability of these classes. By Corollary 10 we get 
Theorem 18. For all $k \in \mathbb{N}_{0}$ we have $\mathcal{M C L S}_{\delta_{\mathrm{h}}}(k) \subset \mathcal{M C} \mathcal{L} \mathcal{S}_{\delta^{*}}(k)$.

Using the set of matching satisfiable multi-clause-sets as studied in [14, the following theorem follows immediately from Theorem 14 and Corollary 12 , matching satisfiable (multi-)clause-sets have been introduced in 7 under the name "matched formulas", and are characterised by the condition $\delta^{*}(F)=0$.

Theorem 19. Consider $F \in \mathcal{M C} \mathcal{L S}_{\delta_{\mathrm{h}}}(1)$.

1. The following three conditions are equivalent:

(a) $F$ is eigensharp

(b) $F$ is matching lean

(c) $\delta(F)=1 \vee F=\top$.

2. $F$ is matching satisfiable iff $\delta(F) \leq 0$.

By Theorem 15 we immediately obtain the following result, which proves Conjecture 16 for all $F \in \mathcal{M C} \mathcal{L S}_{\delta_{\mathrm{h}}}(1)$ (as we will see, this contains the two extremal cases of trees and of complete graphs).

Theorem 20. If the symmetric conflict matrix $A$ is the distance matrix of some graph, and $\delta_{\mathrm{h}}(A) \leq 1$ holds, then $A$ is eigensharp. In terms of multi-clause-sets, consider $F \in \mathcal{M C} \mathcal{L} \mathcal{S}_{\delta_{\mathrm{h}}}(1)$ such that $\mathrm{C}_{\mathrm{s}}(F)$ is the distance matrix of some graph, and $F$ is exact. Then $F$ is eigensharp (and thus $F$ is matching lean, and in case of $F \neq \top$ we have $\delta(F)=1$ ).

Which multi-clause-sets are in $\mathcal{M C} \mathcal{L} \mathcal{S}_{\delta_{\mathrm{h}}}(1)$ ? The general question seems to be fairly complicated, but we will completely characterise the unsatisfiable elements of $\mathcal{M C L S}_{\delta_{\mathrm{h}}}(1)$ in Theorem 26. Before doing so, we consider a special class of matrices with hermitian defect one. A symmetric real matrix $A$ of order $m \in \mathbb{N}_{0}$ is of strictly negative type if for all $x \in \mathbb{R}^{m}, x \neq 0$ with $\sum_{i=1}^{m} x_{i}=0$ we have $x^{t} A x<0$, and $A$ has a zero diagonal. By definition we have, that if $A$ is of strictly negative type, then also every principal submatrix of $A$ is of strictly negative type.

Lemma 21. For every matrix $A$ of strictly negative type we have $\delta_{\mathrm{h}}(A) \leq 1$.

Lemma 22. Consider $F \in \mathcal{M C L S}$, such that $\mathrm{C}_{\mathrm{s}}(F)$ is the distance matrix of a tree, where each edge length can be an arbitrary positive real number. Then $\mathrm{C}_{\mathrm{s}}(F)$ is of strictly negative type (as shown in Corollary 7.2 of [11]), and thus (by Lemma 21) we get $F \in \mathcal{M C L} \mathcal{S}_{\delta_{\mathrm{h}}}(1)$.

It is well known that $J_{m}-I_{m} \in \mathcal{H D}(1)$ for $m \in \mathbb{N}_{0}$ ! 1 Since for any symmetric matrix $A$ and $\lambda \in \mathbb{R}_{>0}$ we have $h(A)=h(\lambda \cdot A)$, it follows that with $A \in \mathcal{H D}(1)$ also $\lambda \cdot A \in \mathcal{H D}(1)$ for every $\lambda \in \mathbb{R}_{>0}$ is the case. ${ }^{2}$ We can generalise these considerations by the following theorem, admitting one "exceptional position".

\footnotetext{
${ }^{1}$ For $m \geq 1$, the matrix $J_{m}-I_{m}$ has the eigenvalue -1 with multiplicity $m-1$ (this follows immediately from $\operatorname{rank}\left(J_{m}\right)=1$; we remind the reader that for symmetric matrices the algebraic multiplicity of an eigenvalue is equal to the geometric multiplicity), while the eigenvalue $m-1$ has multiplicity 1 (just note that $(1, \ldots, 1)^{t}$ is an eigenvector of $\left.J_{m}-I_{m}\right)$.

${ }^{2}$ Perhaps the conceptionally easiest proof is obtained by $h\left(\lambda I_{m}-\lambda J_{m}\right) \geq h\left(\lambda I_{m}\right)-$ $h\left(\lambda J_{m}\right)=m-1$, using Corollary 6 (and $\left.h(A)=h(-A)\right)$.
} 
Theorem 23. For every $\lambda \in \mathbb{R}_{>0}$ and $-\lambda<\mu \leq \lambda$ the matrix $A(\lambda, \mu):=$ $\lambda \cdot\left(J_{m}-I_{m}\right)+\mu \cdot\left(E_{m, p, q}+E_{m, q, p}\right)$ is of strictly negative type for all $m \in \mathbb{N}$ and $1 \leq p<q \leq m$. In general $A(\lambda, \mu)$ is a distance matrix, and in case of $\mu=0$ the matrix $A(\lambda, \mu)$ is the distance of some connected graph (namely the complete graph $K_{m}$ with $m$ nodes).

Lemma 24. For any symmetric matrix $A$ with zero diagonal and of order at least 2 in case there are indices $i \neq j$ with $A_{i, j}=0$ we have $\delta_{\mathrm{h}}(A) \geq 2$.

Lemma 25. Consider $F \in \mathcal{M U S} \mathcal{A} \mathcal{T}_{\delta}(1)$. Then $\mathrm{C}_{\mathrm{s}}(F)$ is a $\{0,1\}$-matrix (i.e., there are no two clauses with two or more clashing literals), and $F \in \mathcal{S M U S} \mathcal{A} \mathcal{T}_{\delta}(1)$ holds if and only if $\mathrm{C}_{\mathrm{s}}(F)=J_{c(F)}-I_{c(F)}$ (i.e., iff every pair of two (different) clauses clash exactly in one literal).

Theorem 26. $\mathcal{M C} \mathcal{L} \mathcal{S}_{\delta_{\mathrm{h}}}(1) \cap \mathcal{U S} \mathcal{A} \mathcal{T}=\mathcal{S} \mathcal{M U S} \mathcal{A} \mathcal{T}_{\delta}(1)$. In words: The unsatisfiable multi-clause-sets with hermitian defect one are exactly the saturated minimally unsatisfiable clause-sets with defect 1.

Corollary 27. Consider $F \in \mathcal{M U S} \mathcal{A} \mathcal{T}_{\delta}(1)$. Then $F$ is eigensharp if and only if $F \in \mathcal{S} \mathcal{M U S} \mathcal{A T}_{\delta}(1)$ holds.

Consider $F \in \mathcal{M U S} \mathcal{A} \mathcal{T}_{\delta}(1) \backslash \mathcal{S} \mathcal{M U S} \mathcal{S} \mathcal{T}_{\delta}(1)$. The question is now whether $F$ is not exact or $\mathrm{C}_{\mathrm{s}}(F)$ is not eigensharp. The simplest example for $F$ is given by $F:=\{\{a\},\{b\},\{\bar{a}, \bar{b}\}\}$. We see that in fact here $\mathrm{C}_{\mathrm{s}}(F)$ is eigensharp, since we have $\mathrm{C}_{\mathrm{s}}(2 \cdot\{v\}+\{\bar{v}\})=\mathrm{C}_{\mathrm{s}}(F)$, and thus $F$ is not exact. Is this the case for all elements of $\mathcal{M U S} \mathcal{S} \mathcal{T}_{\delta}(1) \backslash \mathcal{S} \mathcal{M} \mathcal{U S} \mathcal{A T}_{\delta}(1)$ ? 3

\subsection{Multi-clause-sets with only one positive eigenvalue}

We will see, that $\mathcal{H D}(1)$, the class of symmetric distance matrices $A$ with $\delta_{\mathrm{h}}(A) \leq$ 1 , consists (exactly) of the empty square matrix together with those $A$ with one non-negative eigenvalue, where all other eigenvalues are negative. To this end let us consider the class of symmetric distance matrices $A$, which either are zero matrices or have exactly one positive eigenvalue; more succinctly we can describe this class as the class of all conflict matrices $A$ with $i_{+}(A) \leq$ 1 , and further generalisation for $k \in \mathbb{N}_{0}$, considering multi-clause-sets, yields the classes $\mathcal{M C} \mathcal{L} \mathcal{S}_{i_{+}}(k)$ and $\mathcal{M C} \mathcal{L} \mathcal{S}_{i_{-}}(k)$ where $i_{ \pm}(F):=i_{ \pm}\left(\mathrm{C}_{\mathrm{s}}(F)\right)$. We have $\mathcal{M C L S}_{i_{+}}(0)=\mathcal{M C} \mathcal{L} \mathcal{S}_{i_{-}}(0)=\left\{F \in \mathcal{M C} \mathcal{L S}: \mathrm{C}_{\mathrm{s}}(F)\right.$ is a zero matrix $\}$, and thus $\mathcal{M C} \mathcal{L S}_{ \pm}(0)$ is the set of all $F \in \mathcal{M C L S}$ where every literal is a pure literal for $F$. For every $F \in \mathcal{M C L S}$ we have $h(F) \leq n(F)$, and thus $F \in$ $\mathcal{M C L S}_{i_{+}}(n(F)) \cap \mathcal{M C} \mathcal{L} \mathcal{S}_{i_{-}}(n(F))$. Furthermore

$$
F \in \mathcal{M C} \mathcal{L S}_{i_{+}}(1) \Rightarrow h(F)=i_{-}(F)=\operatorname{rank}\left(\mathrm{C}_{\mathrm{s}}(F)\right)-i_{+}(F)
$$

\footnotetext{
${ }^{3}$ While this paper was in the refereeing process, Xishun Zhao proved that in fact every $F \in \mathcal{M U} \mathcal{S} \mathcal{A} \mathcal{T}_{\delta}(1) \backslash \mathcal{S} \mathcal{M U S} \mathcal{S} \mathcal{T}_{\delta}(1)$ is not exact. And the author realised, that furthermore the class of symmetric conflict matrices of $F \in \mathcal{M U S} \mathcal{A T}_{\delta}(1)$ is exactly the class of adjacency matrices of connected graphs (no parallel edges, no loops), and thus there are $F \in \mathcal{M U S} \mathcal{A T}_{\delta}(1)$ with non-eigensharp symmetric conflict matrix.
} 
holds, where $i_{+}(F)=1$ for $F \neq \top$. By the interlacing inequalities from Subsection 3.3 we get

Lemma 28. If $A$ is a symmetric real matrix, and $B$ is a principal submatrix of $A$, then $i_{+}(B) \leq i_{+}(A)$ and $i_{-}(B) \leq i_{-}(A)$ holds. It follows that for every $k \in \mathbb{N}_{0}$ the classes $\mathcal{M C} \mathcal{L} \mathcal{S}_{i_{+}}(k)$ and $\mathcal{M C} \mathcal{L} \mathcal{S}_{i_{-}}(k)$ are stable under formation of sub-multi-clause-formation and applications of partial assignments.

Since $A \in \mathcal{H D}(1)$ of order at least 2 has no zero eigenvalue, the claim that $A$ has exactly one positive eigenvalue, while all other eigenvalues are negative, reduces to the claim $A \in \mathcal{M C} \mathcal{L S}_{i_{+}}(1)$. We use the Perron-Frobenius theorem now to prove this claim, which needs a bit of preparation. A symmetric conflict matrix $A$ is called irreducible if the graph with adjacency matrix $A$ is connected (see Section 3.2 in [2]), and we call $F \in \mathcal{M C L S}$ irreducible if $\mathrm{C}_{\mathrm{s}}(F)$ is connected. Due to completeness of resolution, every minimally unsatisfiable clause-set is irreducible. If $F \in \mathcal{M C L S}$ does not contain pure literals, then $F$ is not irreducible iff there exist $F_{1}, F_{2} \in \mathcal{M C L} \mathcal{S} \backslash\{\top\}$ with $F=F_{1}+F_{2}$ and $\operatorname{var}\left(F_{1}\right) \cap \operatorname{var}\left(F_{2}\right)=\emptyset$, and thus the assumption, that $F$ is irreducible, is in fact a very natural one (otherwise $F$ is the variable-disjoint union of two non-empty multi-clause-sets). The following theorem is a direct consequence of the Perron-Frobenius theorem (see Theorem 31.8 in 23 , or Theorem 8.8.1 in 8). We remind at the notation $\theta_{i}(A)$ for the eigenvalues of a symmetric real non-empty matrix $A$ in descending order (so that $\theta_{1}(A)$ is the largest eigenvector), and for $F \in \mathcal{M C} \mathcal{L} \mathcal{S}$ we use $\theta_{i}(F):=\theta_{i}\left(\mathrm{C}_{\mathrm{s}}(F)\right)$

Theorem 29. Consider a multi-clause-set $F \in \mathcal{M C L S}$ with $c(F) \neq 0$. For all $1 \leq i \leq c(F)$ we have $\left|\theta_{i}(F)\right| \leq \theta_{1}(F)$. If $F \neq\{\perp\}$, then in fact $\theta_{1}(F)>0$ holds. For any multi-clause-set $T \lesseqgtr F^{\prime} \lesseqgtr F$ we have $\theta_{1}\left(F^{\prime}\right)<\theta_{1}(F)$. If $F$ is irreducible, then $\theta_{1}(F) \geq 0$ has multiplicity one $\left(\theta_{1}(F)\right.$ is a simple root of the characteristic polynomial of $\mathrm{C}_{\mathrm{s}}(F)$ ), and the eigenspace belonging to $\theta_{1}(F)$ is generated by a vector whose coordinates are all positive.

Theorem 30. We have $\mathcal{M C} \mathcal{L} \mathcal{S}_{\delta_{\mathrm{h}}}(1) \subset \mathcal{M C} \mathcal{L} \mathcal{S}_{i_{+}}(1)$.

We conclude this section by an important class of matrices with at most one positive eigenvalue. A square real matrix $A$ of order $m \in \mathbb{N}_{0}$ is of negative type if for all $x \in \mathbb{R}^{m}$ with $\sum_{i=1}^{m} x_{i}=0$ we have $x^{t} A x \leq 0$, and $A$ has a zero diagonal. By definition we have, that if $A$ is of negative type, then also every principal submatrix of $A$ is of negative type.

Lemma 31. For every matrix $A$ of negative type we have $i_{+}(A) \leq 1$.

\section{Hitting clause-sets}

A hitting clause-set is a clause-set where every two (different) clauses clash in at least one literal. We use $\boldsymbol{H \mathcal { I }}$ for the set of all hitting (multi-)clause-sets; note that in a hitting multi-clause-set in fact no clause can occur more than 
once. Obviously we have $F \in \mathcal{H I T} \Leftrightarrow \mathrm{C}_{\mathrm{s}}(F) \geq J_{c(F)}-I_{c(F)}$ (recall example 1 from Section 3). Another basic characterisation of $\mathcal{H I T}$ is that it is the set of (multi-)clause-sets such that there is no partial assignment falsifying two clauses of $F$ at the same time. Some selected properties of $F \in \mathcal{H I T}$ are:

1. For every $\varphi \in \mathcal{P A S S}$ we have $\varphi * F \in \mathcal{H I T}$.

2. For every clause $C$ we have that $F+\{C\} \in \mathcal{H I} \mathcal{I}$ holds iff $\varphi_{C}$ is a satisfying assignment for $F{ }^{4}$

3. $\sum_{C \in F} 2^{-|C|} \leq 1$ and $F \in \mathcal{U S A T} \Leftrightarrow \sum_{C \in F} 2^{-|C|}=1$.

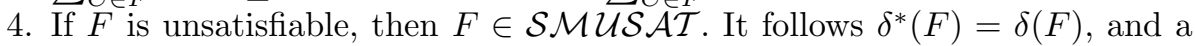
necessary condition for $F \neq\{\perp\}$ being unsatisfiable is that there must exist a variable $v \in \operatorname{var}(F)$ with $\#_{v}(F) \leq \delta(F)$ and $\#_{\bar{v}}(F) \leq \delta(F)$ (see Lemma C.2 in [13]); furthermore we must have $\delta(F) \geq 1$.

Every satisfiable hitting clause-set stays satisfiable when flipping the polarities of arbitrary literal occurrences (see [420] for more on such clause-sets). Let $\mathcal{M U S} \mathcal{A} \mathcal{T}_{k}$ be the set of minimally unsatisfiable clause-sets $F$ such that for every partial assignment $\varphi$ with $n(\varphi) \leq k$ (i.e., $\varphi$ uses at most $k$ variables) also $\varphi * F$ is minimally unsatisfiable; since $F$ is a clause-set, the application of partial assignments contracts multiple occurrences of clauses created by the application, so that for example for any assignment $\varphi$ with $\operatorname{var}(F) \subseteq \operatorname{var}(\varphi)$ the clause-set $\varphi * F$ is minimally unsatisfiable (which for $c(F) \geq 2$ would not be the case for multi-clause-sets $F$ ). By definition we have $\mathcal{M U S} \mathcal{A} \mathcal{T}_{0}=\mathcal{M U S} \mathcal{A} \mathcal{T}$, while in [13] it was shown $\mathcal{M U S} \mathcal{S} \mathcal{T}_{1}=\mathcal{S} \mathcal{M U S} \mathcal{A} \mathcal{T}$.

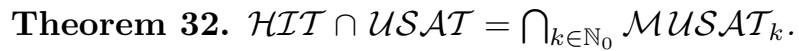

For $k \in \mathbb{N}$ let $\mathcal{U} \mathcal{H} \mathcal{I} \mathcal{T}_{\boldsymbol{k}}$, the set of uniform $k$-hitting clause-sets, be the set of all hitting clause-sets where every two (different) clauses have exactly $k$ conflicts. The set of uniform hitting clause-sets is denoted by $\mathcal{U H \mathcal { H }}:=\bigcup_{k \in \mathbb{N}} \mathcal{U H \mathcal { H }} \mathcal{T}_{k}$. By definition we have $F \in \mathcal{U H} \mathcal{H} \mathcal{I}_{k} \Leftrightarrow \mathrm{C}_{\mathrm{s}}(F)=k \cdot\left(J_{c(F)}-I_{c(F)}\right)$ (recall example 2 from Section 3). $\mathcal{U H} \mathcal{H} \mathcal{I}_{1}$ can be characterised as the class of all clause-sets such that any two (different) clauses have a (non-tautological) resolvent. It is $\mathcal{U H} \mathcal{H} \mathcal{T}_{k}$ stable under application of partial assignments, and for $F \in \mathcal{U H} \mathcal{H} \mathcal{T}_{k}$

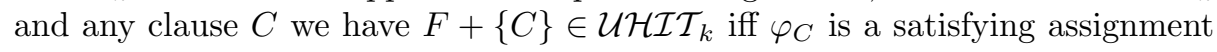
for $F$ which satisfies exactly $k$ literals in every clause of $F$.

Theorem 33. $\mathcal{U H} \mathcal{H} \mathcal{T} \subset \mathcal{M C} \mathcal{L} \mathcal{S}_{\delta_{\mathrm{h}}}(1)$.

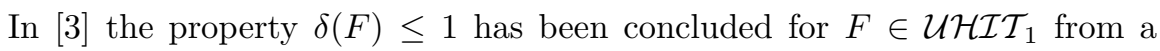
conjecture of Endre Boros; in Section 5 we will give a counterexample for this conjecture, but as we now know, $\delta(F) \leq 1$ in fact must hold (for all $F \in \mathcal{U H} \mathcal{H} \mathcal{I}$ ). The following corollary follows also from the result of $\left[3\right.$ that every $F \in \mathcal{U H} \mathcal{H} \mathcal{T}_{1}$ with $\delta(F) \geq 2$ must be satisfiable (together with known characterisations of minimal unsatisfiable formulas of deficiency 1 ).

\footnotetext{
${ }^{4}$ For a clause $C$ the corresponding partial assignment $\varphi_{C}$ falsifies (exactly) all literals in $C$, while for a partial assignment $\varphi$ the corresponding clause $C_{\varphi}$ consists of all literals falsified by $\varphi$ (partial assignments are finite).
} 
Corollary 34. Consider $F \in \mathcal{U H} \mathcal{H} \mathcal{T}_{k}$ for $k \in \mathbb{N}$. If $k \geq 2$ and $F \neq\{\perp\}$, then $F \in \mathcal{S A T}$. If $k=1$, then $F$ is unsatisfiable iff $F$ is a saturated minimally unsatisfiable clause-set.

\section{On a conjecture of Endre Boros}

At the SAT'1998 workshop in Paderborn, Endre Boros conjectured that every 1uniform hitting clause-set $F$ different from $\{\perp\}$ with defect $\delta(F)=1$ contains a variable $v \in \operatorname{var}(F)$, such that we have $\#_{v}(F)=1$ or $\#_{\bar{v}}(F)=1$. In other words the conjecture states, that every minimum (or exact) biclique decomposition of the complete graph must contain a star (or claw). Now in [19] this question has been discussed in detail, and the smallest counterexample to the conjecture is given by a decomposition of the $K_{9}$ into one $K_{2,4}$ and seven $K_{2,2}$ (that is, one variable has a $(2,4)$-occurrence, while all other variables have $(2,2)$-occurrence). The clause-variable matrix of a corresponding clause-set is

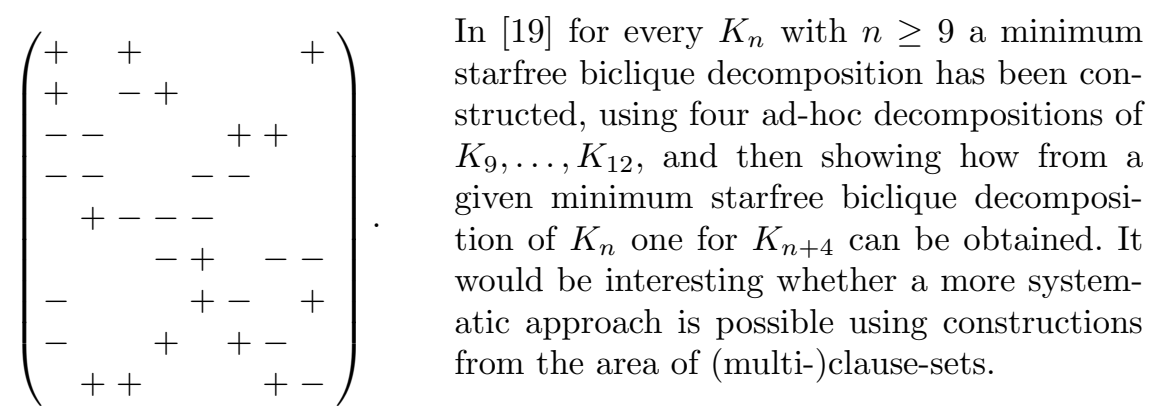

A star-free multi-clause-set has $\#_{v}(F), \#_{\bar{v}}(F) \geq 2$ for all $v \in \operatorname{var}(F)$. Now it is naturally to ask whether for arbitrary $k, \lambda \in \mathbb{N}$ there are eigensharp $k$-uniform hitting clause-sets with $\#_{v}(F), \#_{\bar{v}}(F) \geq \lambda$ for all $v \in \operatorname{var}(F)$ ?!

\section{References}

1. Noga Alon, Richard A. Brualdi, and Bryan L. Shader. Multicolored forests in bipartite decompositions of graphs. Journal of Combinatorial Theory, Series B, 53:143-148, 1991.

2. Richard A. Brualdi and Herbert J. Ryser. Combinatorial Matrix Theory. Cambridge University Press, 1991.

3. Hans Kleine Büning and Xishun Zhao. On the structure of some classes of minimal unsatisfiable formulas. September 2001.

4. Hans Kleine Büning and Xishun Zhao. Satisfiable formulas closed under replacement. In Henry Kautz and Bart Selman, editors, LICS 2001 Workshop on Theory and Applications of Satisfiability Testing (SAT 2001), volume 9 of Electronic Notes in Discrete Mathematics (ENDM). Elsevier Science, June 2001.

5. Randall J. Elzinga, David A. Gregory, and Kevin N. Vander Meulen. Addressing the Petersen graph. Bull. Inst. Math. Acad. Sinicia, 24(2):87-102, 1996. 
6. Herbert Fleischner, Oliver Kullmann, and Stefan Szeider. Polynomial-time recognition of minimal unsatisfiable formulas with fixed clause-variable difference. Theoretical Computer Science, 289(1):503-516, November 2002.

7. John Franco and Allen Van Gelder. A perspective on certain polynomial-time solvable classes of satisfiability. Discrete Applied Mathematics, 125:177-214, 2003.

8. Chris Godsil and Gordon Royle. Algebraic Graph Theory. Springer, 2001.

9. Ronald L. Graham and H.O. Pollak. On the addressing problem for loop switching. Bell System Technical Journal, 50(8):2495-2519, 1971.

10. David A. Gregory, Valerie L. Watts, and Bryan L. Shader. Biclique decompositions and hermitian rank. Linear Algebra and its Applications, 292:267-280, 1999.

11. Poul Hjorth, Petr Lisonĕk, Steen Markvorsen, and Carsten Thomassen. Finite metric spaces of strictly negative type. Linear Algebra and its Applications, 270:255$273,1998$.

12. Thomas Kratzke, Bruce Reznik, and Douglas West. Eigensharp graphs: Decompositions into complete bipartite subgraphs. Transactions of the American Mathematical Society, 308(2):637-653, August 1988.

13. Oliver Kullmann. An application of matroid theory to the SAT problem. In Fifteenth Annual IEEE Conference on Computational Complexity, pages 116-124. IEEE Computer Society, July 2000.

14. Oliver Kullmann. Lean clause-sets: Generalizations of minimally unsatisfiable clause-sets. Discrete Applied Mathematics, 2003. To appear.

15. Oliver Kullmann. On the conflict matrix of clause-sets. Technical Report CSR 7-2003, University of Wales Swansea, Computer Science Report Series, 2003.

16. Oliver Kullmann. Open problems on the combinatorics of conflicts between clauses. Available at http://cs-svr1.swan.ac.uk/ csoliver/Artikel/ OpenProblemsConflicts.html, July 2003.

17. Gábor Kusper. Solving the resolution-free SAT problem by hyper-unit propagation in linear time. In Fifth International Symposium on Theory and Applications of Satisfiability Testing, pages 323-332, 2002.

18. Steven Roman. Advanced Linear Algebra, volume 135 of Graduate Texts in Mathematics. Springer-Verlag, New York, 1992. ISBN 0-387-97837-2; QA184.R65 1992.

19. Allen J. Schwenk and Ping Zhang. Starfree biclique decompositions of complete graphs. Bulletin of the ICA, 23:33-62, 1998.

20. Stefan Szeider. Generalizations of matched CNF formulas. To appear in Annals of Mathematics and Artificial Intelligence, 2002.

21. Stefan Szeider. Minimal unsatisfiable formulas with bounded clause-variable difference are fixed-parameter tractable. Technical Report TR03-002, revision 1, Electronic Colloquium on Computational Complexity (ECCC), February 2003.

22. Jacobus H. van Lint. $\{0,1, *\}$ distance problems in combinatorics. In Ian Anderson, editor, Surveys in Combinatorics, volume 103 of London Mathematical Society Lecture Note Series, pages 113-135. Cambridge University Press, Cambridge, 1985.

23. Jacobus H. van Lint and Richard M. Wilson. A Course in Combinatorics. Cambridge University Press, Cambridge, 1992. ISBN 0521422604. 